\title{
THE ENERGY RESOLUTION AND WINDOW AREA CAPABILITIES OF THE GAS PROPORTIONAL SCINTILLATION COUNTER
}

M. Alice, F. Alves, A.J.P.I. Policarpo and M. Salete S.C.P. Leite Departamento de Física da Universidade de Coimbra Coimbra - Portugal

\section{Abstract}

The behaviour of xenon and argon-xenon gas proportional scintillation counters is con sidered. For X-rays, ranges of window area are defined showing competition with semiconductor detectors. Data for primary scintillation of $\mathrm{X}$-rays and alpha particles are included. Relative efficiencies of tetraphenylbutadiene, p-terphenyl, diphenyl-stilbene, sodium salicylate and p-quaterphenyl were measured.

\section{Introduction}

The gas proportional scintillation counter is essentially a gaseous device where scin tillations are generated, its light output being controlable by application of suitable electric fields. The main lines of research are summarized by P. E. Thiess and G.H. Mi ley $^{1}$, most of the work done till now being on nuclear physics applications, as a detector, both in low and high radiation fields.

As a detector, the scintillation is generated by an ionizing particle and essentially two main components are considered in the corresponding light flash: the primary scintillation due to the deexcitation of the medium following the interaction of the particle being detected; and the secondary scintillation corresponding to the light emitted during the migration of the secondary electrons under electric fields. Both the primary and the secondary components have been studied for a wide range of gas mixtures ${ }^{1}$. In particular, for $X$-ray detection, probably the best way to study the main statistical factors relevant to the behaviour of the gas proportional scintillation counter, the energy resolution and counting rate capabilities associated with the secondary component have been considered $^{2}, 3,4,5$.

The window area is also an important parameter. Provided that a reasonable degree of localization is attained for the region where the secondary light is produced, and because the electronic noise and detector capacitance play no role on the behaviour of the gas proportional scintillation counter, it is expected that large window areas can be used without deterioration of the resolution 2,3 .

The gas proportional scintillation counter may compete with the semiconductor $\mathrm{x}$-ray detectors as far as energy resolution is concerned, if the window area parameter is taken into account. Criteria related to system resolution and detector area, for cooled silicon $\mathrm{X}$-ray detectors were analised by R. S. Frankel and D. W. Aitken ${ }^{6}$ and areas till about $500 \mathrm{~mm}^{2}$ may be very useful. It was then decided to study the variation of the resolution with window area for the gas proportional scintilla tion counter on the same region of window areas.

Another important parameter relevant for the energy resolution of the gas proportional scintillation counter is the light output of the detector per unit of energy, under condi- tions of no charge multiplication; and this is essentially determined by the nature of the wavelength shifters used and the gas filling. Concerning the primary scintillation an exten sive study was made by J.A. Northrop and J. C. Gursky ${ }^{7}$ and pure xenon and mixtures of neon-508 xenon and helium-10\% xenon have the greatest light output, when diphenylstilbene and a glass window photomultiplier tube are used. But when one tries to choose a mixture for gas proportional scintillation counters the available data concerning light outputs are scantier, the main reason being that most of the studies have been made in conditions of charge multiplication, which means that they are not a valid indication of the amount of secondary light per electron. Under conditions of charge multiplication pure xenon, helium-10\% xenon, and argon-5\% xenon seem to be the best scintillators on the ultraviolet region 8,9 ; if the visible and infrared regions are included then mixtures of $\mathrm{Ne}-\mathrm{He}, \mathrm{Ne}-\mathrm{Ar}, \mathrm{Ne}-\mathrm{O}_{2}, \mathrm{He}-\mathrm{N}_{2}, \mathrm{He}-\mathrm{O}_{2}$, Ar $-\mathrm{N}_{2}$ and pure helium and argon are also very good scintillators ${ }^{l}$. In view of previous experience of the authors and the available data, xenon and argon $-5 \%$ xenon were used as filling gases and the ultraviolet emission was detected through the use of wavelength shifters and quartz window photomultipliers, EMI 9656QR.

Again most of the available data for wave length shifters efficiencies concern either the primary scintillation or the scintillation on conditions of charge multiplication. Then a previous study ${ }^{10}$ of $\mathrm{p}$-terphenyl, sodium salicy late and p-quaterphenyl, as wavelength shifters for xenon proportional scintillation coun ters under conditions of no charge multiplication, is continued using $1,1,4,4$, tetraphenylbutadiene and 4,4' diphenylstilbene.

\section{Experimental techniques}

Fig. I shows the detector used in this work. The main concern was to make it mechanically strong, so no magnesium oxide reflector was used. For a detailed description of the de tecting system, associated electronics and $\mathrm{x}-$ -ray generation see references 2 and 3 . The pu rities of the gases used, xenon and argon commercially available, are, respectively, $99.95 \%$ (impurities: $\mathrm{Kr}<400 \mathrm{v} \cdot \mathrm{p} . \mathrm{m} ., \mathrm{N}_{2}<10 \mathrm{v} \cdot \mathrm{p} . \mathrm{m} ., \mathrm{O}_{2}<5$ v.p.m., $\mathrm{H}_{2} \mathrm{O}<5 \mathrm{v} \cdot \mathrm{p} \cdot \mathrm{m} ., \mathrm{CO}<5 \mathrm{v} \cdot \mathrm{p} . \mathrm{m} ., \mathrm{CO}_{2}<5 \mathrm{v} \cdot \mathrm{p} \cdot \mathrm{m}$ $\mathrm{CH}_{4}<5$ v.p.m.) and $99.9 \%$ (humidity $<10 \mathrm{mg} / \mathrm{m}^{3}$ ), and they were continuously purified.

As in a previous work ${ }^{10}$, to study efficiencies of wavelength shifters and in order to avoid reflections, no aluminium reflector was used, one of the quartz windows was replaced by a stainless steel cover and the inside face of the other was masked by a stainless steel plate with a central circular aperture (diameter $29 \mathrm{~mm}$ ) where a thin quartz disc with the wavelength shifter deposit could be placed. The thicknesses of the deposits were de- 
termined by weighting.

To compare the light output of xenon and the promising mixture argon-58 xenon, and to avoid corrections due to the different riseti mes of the pulses, dependent on the gas filling nature and the applied voltage (the pres sure was kept constant), no differentiation was introduced on the amplifiers, an integrating time constant of 100 ns was used and, in order to match the characteristics of the pul se height analyser the pulses were fed through a linear gate. The charge pulses were analysed on the same way.

Work related to primary scintillations was carried on taking out the anode and teflon insulator. When alpha particles from a 'ThC+ThC' source were used the entrance window was made of mylar $6 \mu \mathrm{m}$ thick, the beam was well collimated, and a vacuum path was created between the source and the mylar window to minimize straggling. In each photomultipli er three dynodes were short-circuited to the anode to avoid saturations.

To study the variation of the energy resolution with window area a rectangular aperture of $34 \times 18 \mathrm{~mm}$ was made in the stainless steel body of the counter; windows, framed in stainless steel, were then screwed into place with indium gaskets. The alpha source and the targets for $\mathrm{X}$-ray generation were located as far away as possible from the entrance win dow, in order to get a relatively uniform flux of $X$-ray over the entire window area.

\section{Experimental results and conclusions}

\section{Wavelength shifters}

Following a previous work ${ }^{10}, 1,1,4,4$ tetraphenylbutadiene and 4,4' diphenylstilbene, both NE scintillation grade, were studied in the xenon gas scintillation counter at a pressure of 940 Torr and an anode voltege of $5000 \mathrm{~V}$ (ionization chamber region).

$\mathrm{X}$-rays from a ${ }^{55} \mathrm{Fe}$ source were used and the mean light pulse amplitude $h$ was measured as a function of the thickness $t$ of the wavelength shifter. In view of the conclusion of a previous work ${ }^{10}$ together with some measurements for tetraphenylbutadiene and diphenylstilbene we consider that the data obtained at 5000 volts are representative of the behaviour of the wavelength shifter for any anode voltage down to about 2000 volts. To be able to compare this data with the data gathered before, p-ierphenyl scintillation grade at its optimum thickness was used for normalization.

For each wavelength shifter the thickness $t_{M}$ of maximum light output $h_{M}$ and the minimum thickness $t_{m}$ for which $0.9 h_{M}=h\left(t_{m}\right)$ are $t a-$ bulated, as well as the relative efficiencies at optimum thickness:

\begin{tabular}{|l|c|c|c|}
\hline $\begin{array}{l}\text { Wavelength } \\
\text { shifter }\end{array}$ & $\begin{array}{c}t_{\mathrm{M}} \\
\left(\mathrm{mg} / \mathrm{cm}^{2}\right)\end{array}$ & $\begin{array}{c}t_{\mathrm{m}} \\
\left(\mathrm{mg} / \mathrm{cm}^{2}\right)\end{array}$ & $\begin{array}{c}\text { relative } \\
\text { efficiency }\end{array}$ \\
\hline p-terphenyl & 2.5 & .55 & 1 \\
tetraphenyl- & 2.5 & .20 & 1 \\
butadiene & .25 & .15 & .9 \\
diphenylstilbene & .45 & .7 \\
Na salicylate & 1.0 & .22 & .6 \\
\hline p-quaterphenyl & .28 & .22 & \\
\hline
\end{tabular}

In view of these results and taking into account information relative to possible deteri oration of the tetraphenylbutadiene deposits ${ }^{\overline{1}}$, p-terphenyl was used as wavelength shifter.

\section{Xenon and argon -58 xenon}

Fig. 2 shows the secondary light outputs, $L$, from pure xenon and an argon-5\% xenon mixture for aluminium $\mathrm{x}$-rays, as a function of the anode voltage, using p-terphenyl as wavelength shifter. The anode was a lomm diameter sphere and the pressure 970 torr. Charge measurements using alpha particles showed that for the range of voltages used there was no charge multiplication when xenon was the filling gas; the dashed line in the same fig. shows the charge gain, as a function of the voltage, for the argon-5\% xenon mixture.

The threshold for light multiplication is much lower for the mixture than for pure xenon, and for voltages below 1400 volts xenon is a poorer scintillator". Again for voltages over 3000 volts xenon gives a lower light output. But, as the charge curve shows, this effect is associated with the charge multiplication process. This explains the data previously reported 8,9 , working under conditions of large charge gains, and quoting light outputs from the mixture an order of magnitude larger than those from pure xenon. If the resolution is the important parameter of the detector, and this depends on the particular application, the relevant parameter is the light output below the charge multiplication threshold, or rather, $d L / d V$ on the ionization chamber region. In this context xenon is a better scintillator than the mixture argon-5\% xenon. These results were further confirmed using a $6 \mathrm{~mm}$ dia meter anode.

Xenon was then the filling gas choosen for the proportional scintillation counter.

\section{Primary scintillation}

In order to show the optical efficiency of the counter used in these experiments, primary scintillation spectra from silver $x$-rays and ThC+ThC' alpha particles are represented in fig. 3. The higher energy alpha peak corres ponds to about $8.3 \mathrm{MeV}$ dissipated in the detector. If this energy loss is taken into account the detector has the same optical efficiency as the gas scintillation counter of Say res and $\mathrm{Wu}^{12}$, which features the best resolution reported. The $X$-ray spectra in the same fig. illustrates the possibility of detecting the primary scintillations of relatively low energies well above the noise.

\section{X-ray energy resolution and window area}

Fig. 4 shows spectra for carbon, aluminium and manganese $\mathrm{K} \alpha \mathrm{X}$-rays resulting from the bombardment of graphite and aluminium targets with alpha particles and from a

55

source with a chromium filter. The corres ponding energies are $0.277 \mathrm{keV}, 1.49 \mathrm{keV}$ and $5.9 \mathrm{keV}$, respectively, and the conditions of pressure, applied voltage and the diameter of the spherical anode are quoted as well as the resolutions and widths at half maximum. The filling gas was xenon, continuously purified, and the wavelength shifter used was p-terphenyl with a thickness of about $2.5 \mathrm{mg} / \mathrm{cm}^{2}$. From 
this type of data one arrives to the expression $\sigma_{r}=0.081 \mathrm{E}^{-1 / 2}(\mathrm{E}$ in $\mathrm{keV})$, relating the relative standard deviation with the energy. Aluminium $X$-rays were used to study the variation of the resolution with window area. Ten different sizes of window were used, circu lar and rectangular, covering a region between 16 and $480 \mathrm{~mm}^{2}$. Within experimental uncertainties, of the order of $4 \%$, no variation of reso lution with window area was detected, as should be expected. To compare the xenon gas proportional scintillation counter with silicon $\mathrm{X}$-ray detectors, as far as resolution and window area are concerned, a compilation of data is represented in Fig. 5 for aluminium $X$-rays and in Fig. 6 for oxygen and carbon X-rays. The shaded areas are regions of typical resolutions of silicon detectors, based on available data from two well known manufacturers for the ener gy resolution at $5.898 \mathrm{keV}$, the manganese $\mathrm{K}_{\alpha}$

$\mathrm{X}$-rays, at low counting rates, for several detector dimensions. It was assumed that the electronic noise is independent of the energy dissipated in the detector and 0.1 was taken as the Fano factor for silicon. In Fig. 5 the band of window areas is limited by the data available; for a certain window area and a cer tain shaded region, the upper and lower resolu tion limits correspond to the use of resistor or pulsed optical feedback. Fig. 6 is similar, but of course, there is no sense in considering f.w.h.m. that would make the detector inoperative on account of noise counting rates, and the shaded regions were limited by full widths at half maximum of the order of a third of the $\mathrm{X}$-ray energy. The regions so obtained for silicon detectors were confirmed to be rea sonably correct by comparing with experimental data available for each of the $x$-rays considered. In particular, and due to its interest, experimental data are represented for carbon $\mathrm{X}$-rays using silicon detectors (see Fig. 6), corresponding to the results of J.M. Jaklevic and F.S. Goulding ${ }^{13}$ and R.G. Musket and W. Bauer $^{14}$.

The dashed lines in Figs. 5 and 6 correspond to the experimental energy resolutions of the xenon proportional scintillation counter as given by $\sigma_{r}=0.081 \mathrm{E}^{-1 / 2}$ ( $\mathrm{E}$ in $\left.\mathrm{keV}\right)$.

From Fig. 5 one may infer the practical conclusion that for window areas below about $25 \mathrm{~mm}^{2}$ the silicon $\mathrm{x}$-rays detector has better resolution than the xenon gas proportional scintillation counter; between 25 and $190 \mathrm{~mm}^{2}$ there is competition between both types of detectors; and the xenon counter dominates above $190 \mathrm{~mm}^{2}$. The energy resolution of silicon detectors varies with counting rate as the electronic noise depends on the time constants used. The insert of Fig. 5 shows for several time constants, a typical variation of the $f$. w.h.m. with detector area for aluminium X-rays in $\mathrm{Si}(\mathrm{Li}$ ) detectors (full lines) and in the $\mathrm{Xe}$ PS counter (dashed line). Again those curves were calculated from data available for
5.89 and $6.40 \mathrm{keV} \mathrm{X}$-rays ${ }^{6}$. Then, in Figs. 5 and 6 the effect of higher counting rate would be a shift upwards of the shaded areas that correspond to silicon detectors; and recalling the previous example of the aluminium $\mathrm{X}$-rays (see Fig. 5) one may take the band between $25 \mathrm{~mm}^{2}$ and $150 \mathrm{~mm}^{2}$ as the region of competition between silicon detectors and the Xe PS counter.

From this type of data emerges Fig. 7: the shaded area is a region of competition between semiconductor and xenon detectors as far as energy resolution and window area are concerned; the left side region corresponds to a better performance for silicon detectors; the right side region to a better performance of the xenon proportional scintillation counter.

We are indebted to Professor J.R. de Almeida Santos for his continuous encouragement.

Financial support given by Instituto de Alta Cultura (Project CF/2) is gratefully aknowledged.

\section{References}

1 - P.E. Thiess and G.M. Miley, IEEE Trans. Nucl Sci. NS-21, no 1 (1974) 125.

2 - A.J.P.L. Policarpo, M.A.F. Alves, M.C.M. dos Santos and M.J.T. Carvalho, Nucl. Instr. Meth. 102 (1972) 337.

3 - A.J.P.L. Policarpo, M.A.F. Alves, M. Salete S.C.P. Leite and M.C:M. dos Santos, Nucl. Instr. Meth. 118 (1974) 221.

4 - M.A.F. Alves, A.J.P.L. Policarpo and M.C. M. dos Santos, Nucl. Instr. Meth. 111 (1973) 413.

5 - H.E. Palmer and L.A. Braby, Nucl. Instr. Meth. 116 (1974) 587.

6 - R.S. Fkankel and D.W. Aitken, in "Applications of low energy $X$ and gamma rays" ed. C.A. Ziegler; Gordon and Breach, N. Y. $1971,335$.

7 - J.A. Northrop and J.C. Gursky, Nucl. Instr. 3 (1958) 207.

8 - A.J.P.L. Policarpo, C.A.N. Conde and M.A. F. Alves, Nucl. Instr. Meth 58 (1968) 151.

9 - I.B. Keirim-Markus, A.K. Savinskii, V.G. Chaikovskii and A.S. Yakovlev, Instr. Exp. Tech. 15 (Spt-Oct. 1972) 1337.

10- M.A.F. Alves, M.C.M. dos Santos and A.J.P. L. Policarpo, Nucl. Instr. Meth. 119 (1974) 405 .

11-M. Brunet, M. Cantin, C. Julliot and J. Vasseur, J. Phys. (Phys. Appl.) Suppl. au no 3, 24 (Mars 1963) 53A.

12- A. Sayres and C.S. Wu, Rev. Sci. Instr. 28 (1957) 758 .

13- J.M. Jaklevic and F.S. Goulding, IEEE Trans Nucl. Sci. NS-18, no 1 (1971) 187.

14- R.G. Musket and W. Bauer, in "X-ray energy spectrometry" R. Woldseth, Kevex Corp. (1973) 1.20 . 

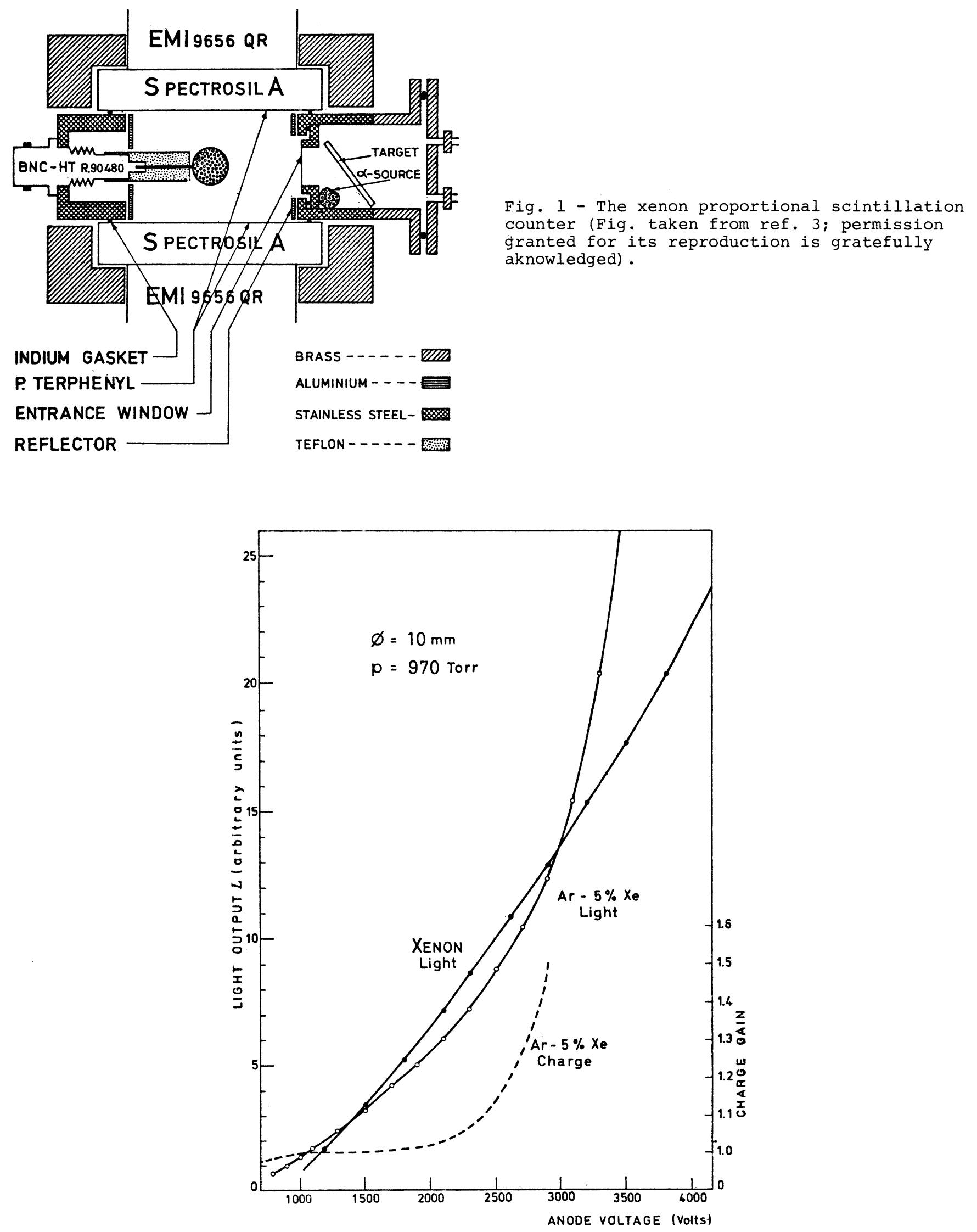

Fig. 2 - Light output from xenon (full circles) and from argon-5\% xenon mixture (open circles) as a function of the anode voltage. The dashed line (right hand scale) represents the charge gain vs. anode voltage for the Ar-5\% Xe mixture. 


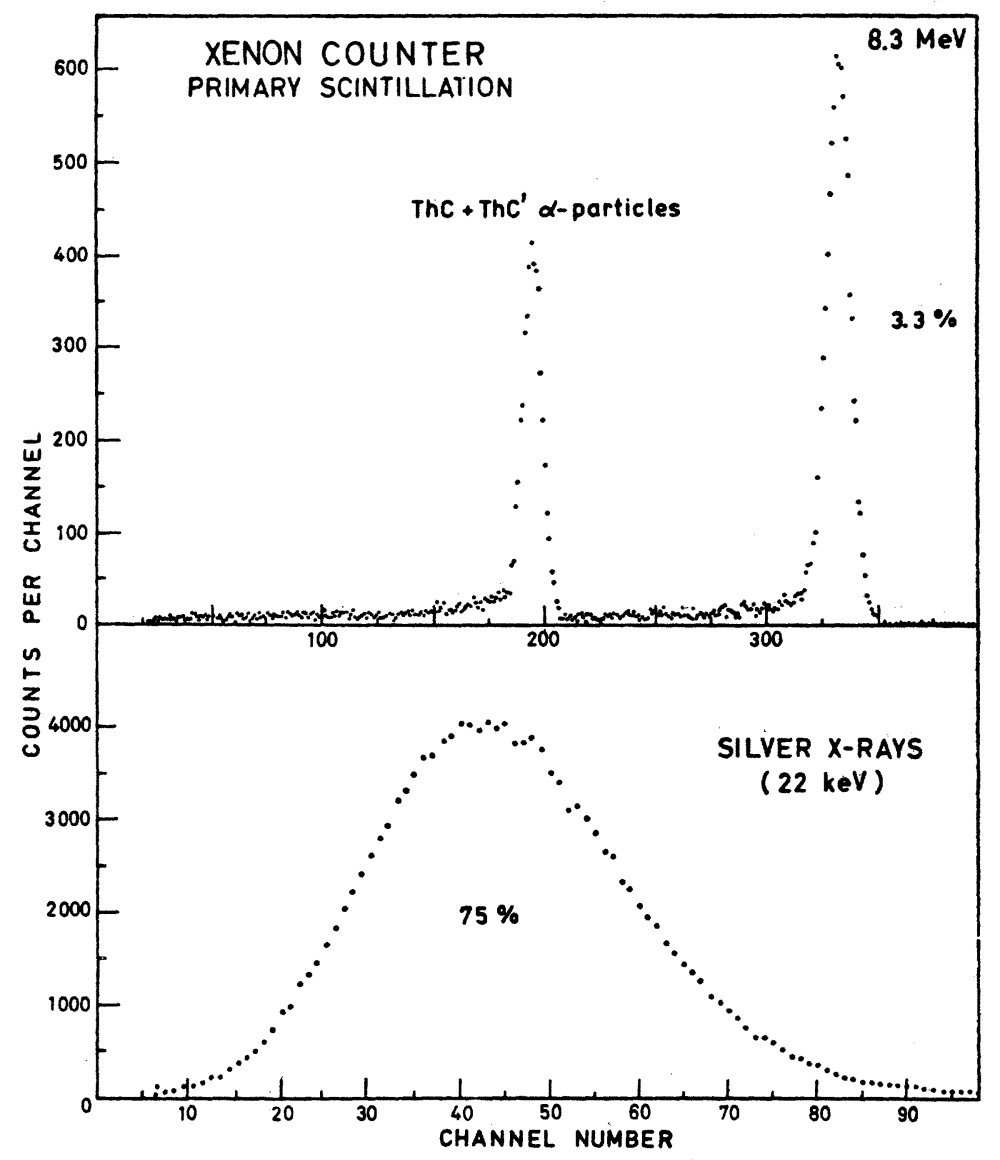

Fig. 3 - Primary scintillation spectra of

ThC+ThC' alpha particles and of silver X-rays

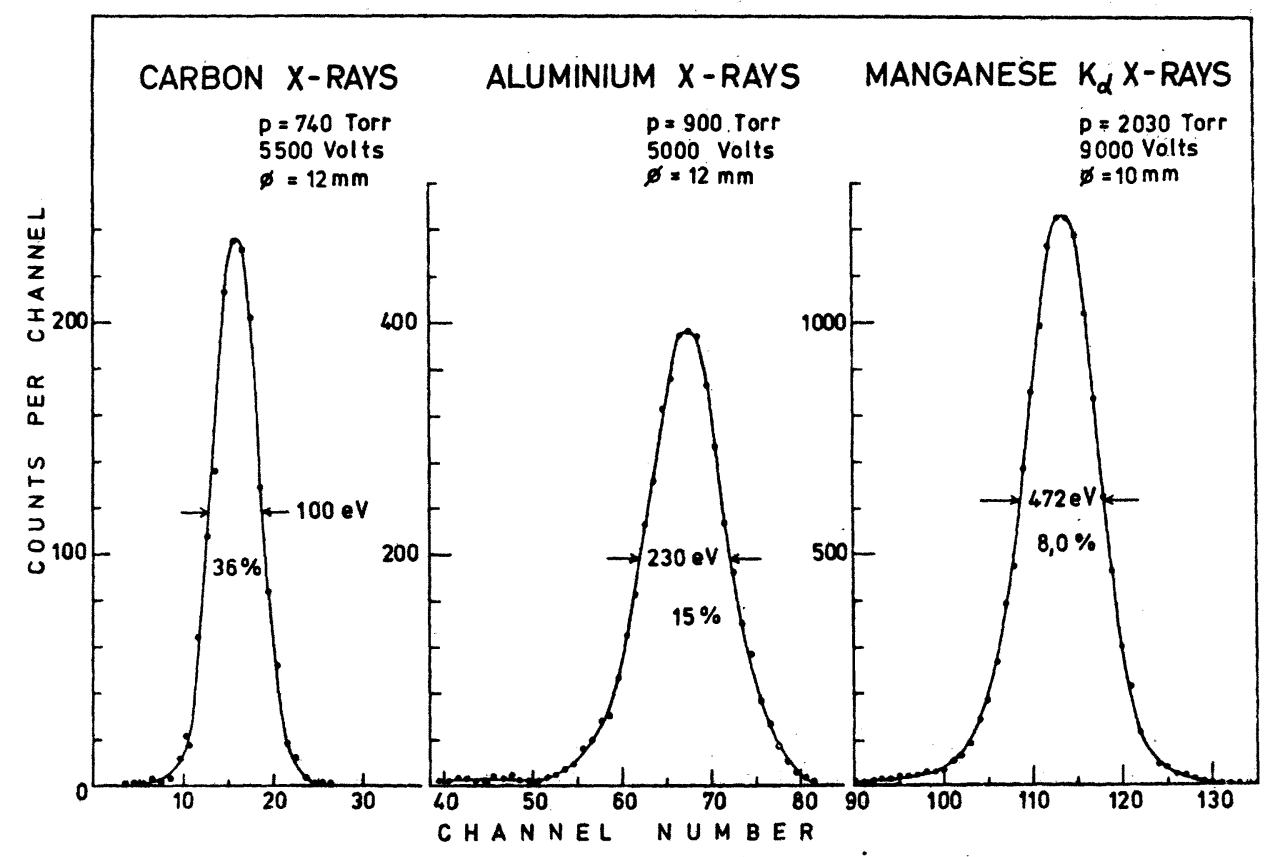

Fig. 4 - Spectra of carbon (277 ev), aluminium ( $1.49 \mathrm{keV})$ and manganese $K_{\alpha}(5.898 \mathrm{keV})$ $\mathrm{x}$-rays obtained with the xenon proportional scintillation counter. 


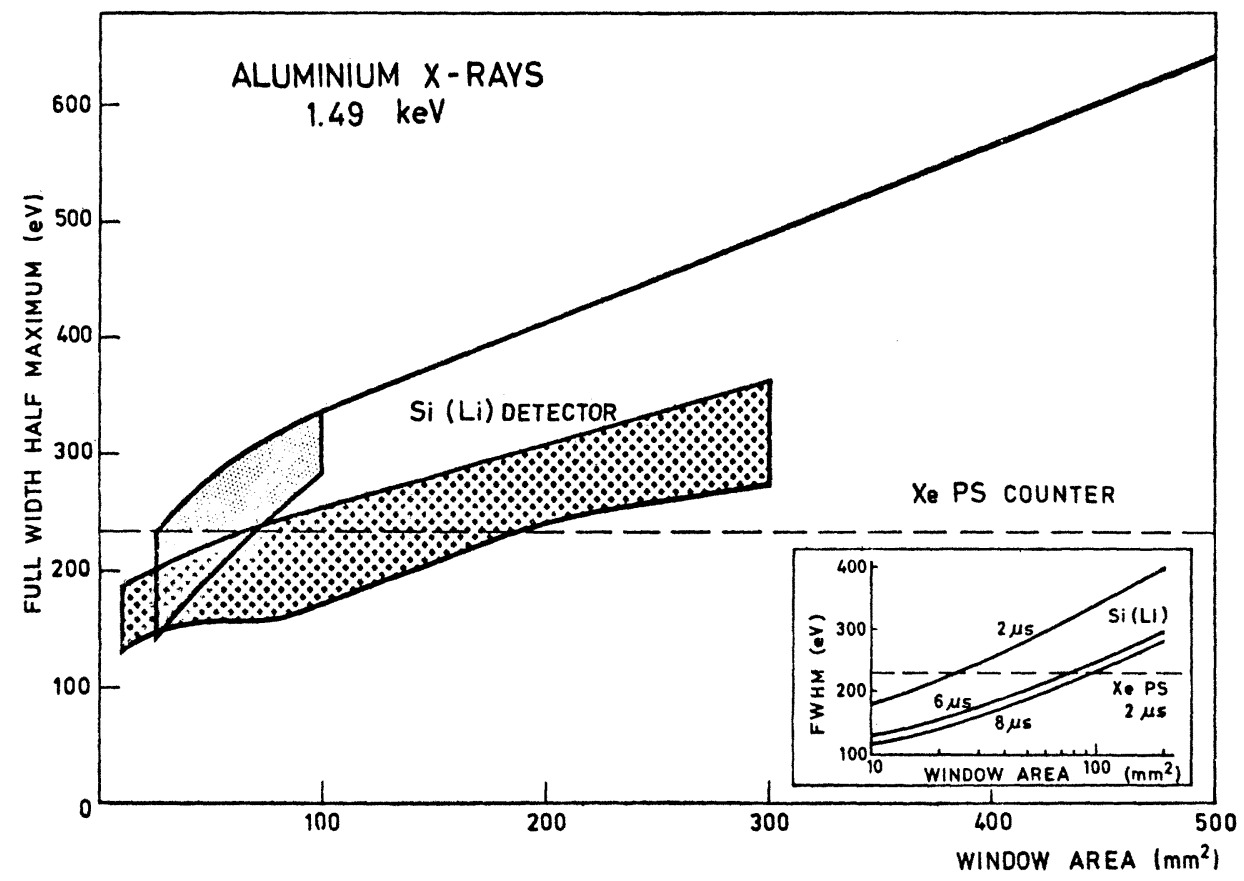

Fig. 5 - Full lines and shaded areas correspond to typical values of f.w.h.m. for 1.49 $\mathrm{keV}$ in $\mathrm{Si}(\mathrm{Li})$ detectors (see text); dashed line represents experimental f.w.h.m. of the xenon proportional scintillation counter for the same energy.

The insert shows typical f.w.h.m. for Al $x$-rays as a function of window area and time constants both for $S i(\mathrm{~L} i$ ) detectors (full lines) and for Xe PS counter (dashed line). 


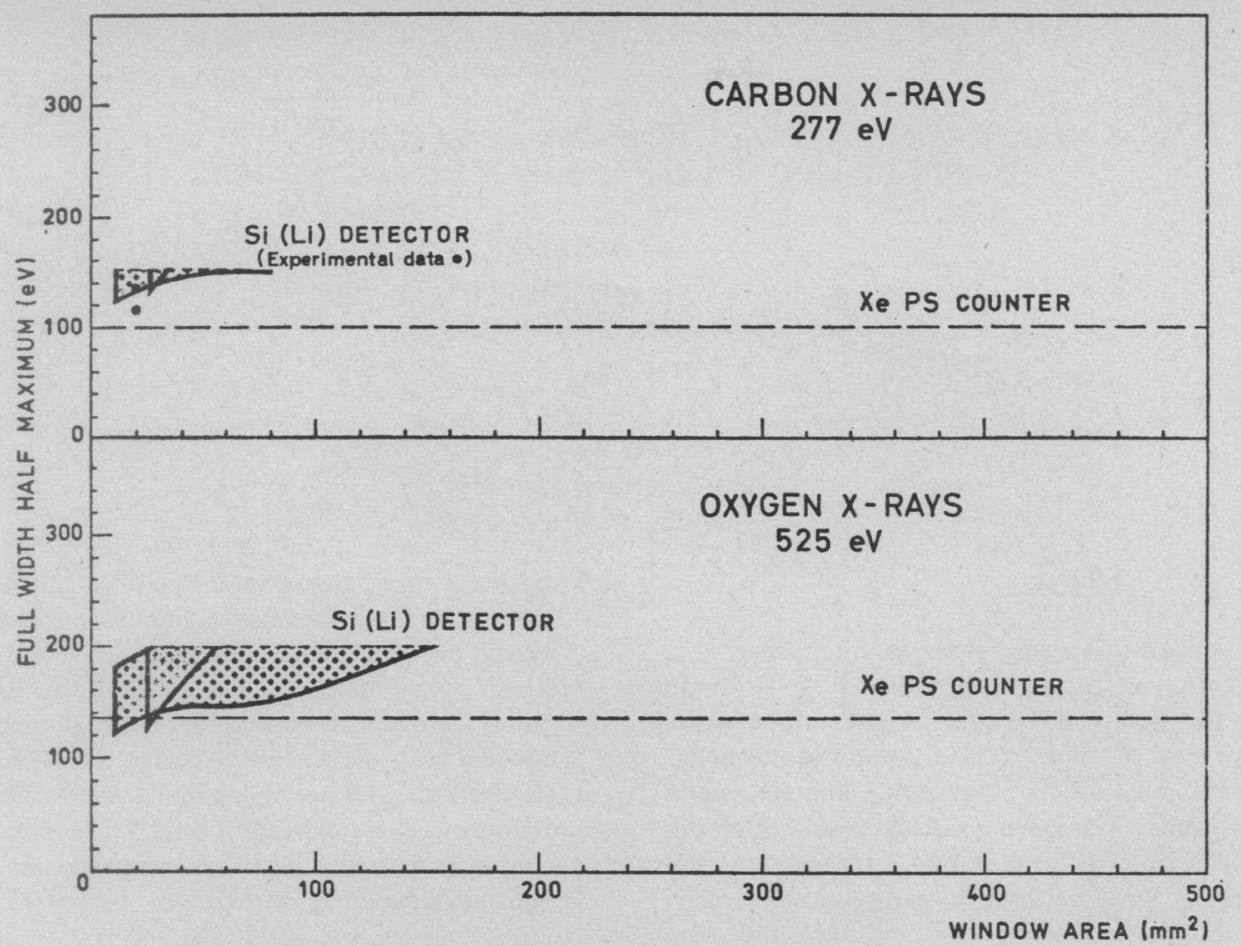

Fig. 6 - Full lines and dashed areas correspond to typical values of f.w.h.m. for $277 \mathrm{eV}$ and for $525 \mathrm{eV}$ in Si(Li) detectors (see text). The dashed lines represent the experimental f.w.h.m. of the xenon proportional scintillation counter for carbon and oxygen X-rays. Full circles are experimental values taken from refs. 13 and 14 .

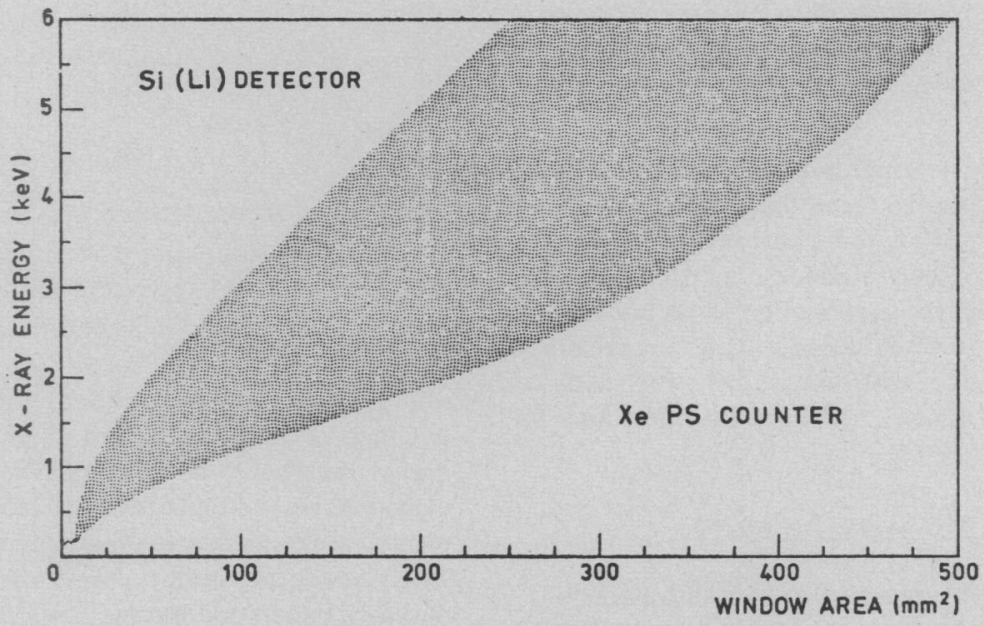

Fig. 7 - Comparative performance of semiconductor and xenon proportional scintillation detectors when the energy and window area vary within the ranges shown. The shaded area represents the region where both detectors are competitive; the left side region corres ponds to a better performance of $\mathrm{Si}(\mathrm{Li})$ detẹ tors and the right side to a better performan ce of Xe PS counters. 